IDDF2018-ABS-0204 EFFECT OF WHOLE GRAINS AND UNSATURATED FATTY ACIDS DIETARY INTERVENTION ON HEALTH IN HONG KONG OVERWEIGHT POPULATION

Christopher Pui Sing Ng*, Jamie Sui Lam Kwok, Junwei Zhou, Pik Yuen Belinda Cheung, Lawrence Chi Hung Tzang, Senthil Sundaram. Prenetics Limited, Hong Kong

\subsection{6/gutjn|-2018-IDDFabstracts. 153}

Background To determine the effect of different dietary interventions on health outcomes in the Hong Kong East Asian population.

Methods 38 overweight or obese (BMI $>23 \mathrm{~kg} / \mathrm{m}^{2}$ ) East Asian subjects ( 25 to 60 years of age, 19 males, 19 females) from Hong Kong were recruited as part of a randomised controlled clinical trial. People with major pulmonary, cardiovascular, hepatic, gastrointestinal or renal diseases, metabolic syndrome or recent antibiotic use were excluded. All subjects were randomly assigned four diet groups, 1) Diet as usual (control), 2) Unsaturated fatty acid diet (UFA), 3) Whole grain diet (WG), 4) Mixed intervention of 2nd and 3rd diets (MIX). Interventions were provided by a registered dietitian. A separate dietitian who was blinded monitored the compliance to the assigned diet type by visual assessment of food consumption. To determine the health outcomes, physical examination, blood pressure, blood glucose and serum cholesterol were assessed at the body check centre at week 0 (baseline), 1, 4 and 8. Dietary interventions were compared with controls using ttest.

Results A total of 38 subjects observed throughout the 8 weeks, 11 subjects were randomly allocated to WG group, 10 to PUFA, 8 to MIX and 7 to the control group. Across all 4 diet groups, overall compliance was $25 \%$, with an $11 \%$ increase at week 8 compared to baseline. Compliance to dietary intervention had a favourable effect on total and LDL cholesterol levels. A negative correlation between compliance change versus total and LDL cholesterol change at week 8 compared to baseline were observed $(r=-0.4, \quad r=-0.41$ respectively). Compared to controls, a significant reduction of systolic blood pressure by 8 th week was observed in UFA group $(p=0.0048)$, unremarkable in other groups. A trend towards significant reduction of triglycerides was observed by 8th week $(p=0.059)$ in WG group compared to controls. Both MIX and WG groups showed a trend towards reduction of total cholesterol $(p=0.09$ and $p=0.05$ respectively).

Conclusions Effect of dietary intervention on health depends on the specific interventions. High unsaturated fatty acid diet has a favourable effect on reducing systolic blood pressure.

\section{IDDF2018-ABS-0206 THE IMPACT AND EFFECTIVENESS OF IBS CLINICAL PATHWAY IN OUTPATIENT MANAGEMENT OF IRRITABLE BOWEL SYNDROME IN A TERTIARY CENTRE}

${ }^{1}$ Dian Farhan Muhamad Sarkan*, 'Deborah Chia Hsin Chew, ${ }^{1}$ Zhiqin Wong, ${ }^{1}$ Chai Soon Ngiu, ${ }^{2}$ Azniza Ismail, ${ }^{3}$ Yeong Yeh Lee, ${ }^{1}$ Raja Affendi Raja Ali. ' Gastroenterology Unit, Faculty of Medicine, National University of Malaysia Medical Centre, Kuala Lumpur, Malaysia; ${ }^{2}$ Department of Community Medicine, Faculty of Medicine, The National University of Malaysia, Kuala Lumpur, Malaysia; ${ }^{3}$ Gastroenterology Unit, Faculty of Medicine, University of Science Malaysia, Kubang Kerian, Kelantan, Malaysia

\subsection{6/gutjnl-2018-IDDFabstracts. 154}

Background Irritable bowel syndrome (IBS) is a chronic gastrointestinal disorder consisting of abdominal pain and altered bowel habit with either diarrhoea, constipation or both which has a significant impact on patient's quality of life. To date, there have been no published studies on the efficacy of a clinical pathway in treating IBS. The IBS Clinical Pathway (CP) consists of an algorithm that delivers standardised care and prompt intervention to IBS patients. We aim to assess the effectiveness of IBS clinical pathway in improving symptoms and severity of IBS.

Methods This was a non-randomised prospective study in Universiti Kebangsaan Malaysia Medical Centre (UKMMC) from June 2017 to May 2018. We included all patients that fulfilled the diagnosis of IBS based on Rome III criteria. The patients from CP group and non-CP group had their baseline IBS severity scoring system (IVS-SSS), Euro quality of life 5dimension 5-levels (EQ-VAS) and hospital anxiety and depression scale (HADS) score calculated at baseline and the end of the study. CP followed a strict algorithm from diagnosis consisting of dietary counselling for low FODMAP diet during each visit, close biopsychosocial assessment and those with moderate to severe HADS scores were referred to psychiatry for appropriate intervention.

Results 101 patients were recruited under CP and 84 patients for the non-CP group. At the end of the study, the CP group demonstrated a statistically significant improvement in their IBS Severity Score: 20 participants (19.8\%) showed significant IBS Severity Score improvement in CP group whereas only 7 participants $(8.3 \%)$ in non-CP group $(p=0.006)$ demonstrated an improvement. For EQ-VAS score, both groups showed significant improvement in the level of health at the end of the study ( $p$ value $<0.05$ ). There was a significant improvement in IBS symptoms with prompt psychiatry referral in CP groups $(p=0.024)$ but not in non-CP group $(p=0.171)$. In relation between IBS severity improvement and low FODMAP diet, there were no significant difference in both groups $(p>0.05)$. Conclusions IBS Clinical Pathway is effective in improving IBS severity and symptoms and should be incorporated into clinical practice.

\section{IDDF2018-ABS-0209 VENOUS THROMBOEMBOLISM IN PATIENTS WITH INFLAMMATORY BOWEL DISEASE IN CHINA: INVESTIGATIONS INTO INCIDENCE AND RISK FACTORS}

${ }^{1}$ Jing Liu*, ${ }^{2}$ Xiang Gao, ${ }^{3}$ Ye Chen, ${ }^{4}$ Qiao Mei, ${ }^{5}$ Liangru Zhu, ${ }^{6}$ Jiaming Qian, ${ }^{2}$ Pinjin Hu. ${ }^{1}$ Inflammatory Bowel Disease Center, Sir Run Run Shaw Hospital, Zhejiang University School of Medicine, China; ${ }^{2}$ Department of Gastroenterology, The Sixth Affiliated Hospital of Sun Yat-sen University, China; ${ }^{3}$ Department of Gastroenterology, Nanfang Hospital of Southern Medical University, China; ${ }^{4}$ Department of Gastroenterology, The First Affiliated Hospital of Anhui Medical University, China; ${ }^{5}$ Department of Gastroenterology, Union Hospital, Tongji Medical College, Huazhong University of Science and Technology, China; ${ }^{6}$ Department of Gastroenterology, Peking Union Medical College Hospital, China

\subsection{6/gutjnl-2018-IDDFabstracts.155}

Background Studies from the western population proved inflammatory bowel disease (IBD) a risk factor for venous thromboembolism (VTE) and thromboprophylaxis has become standard care for hospitalised IBD patients with disease flares. In Asia, lack of studies on VTE among IBD patients makes practice of thromboprophylaxis far from standardised. Our aim is to explore the incidence and risk factors for VTE among Chinese IBD patients.

Methods Retrospective analysis of IBD patients from 17 Chinese tertiary referral hospitals between 2011 and 2016 was 